TITLE:

\title{
Uplink Contention-Based CSI Feedback with Prioritized Layers for a Multi-Carrier System
}

\author{
AUTHOR(S): \\ Kaneko, Megumi; Hayashi, Kazunori; Popovski, \\ Petar; Yomo, Hiroyuki; Sakai, Hideaki
}

\section{CITATION:}

Kaneko, Megumi ...[et al]. Uplink Contention-Based CSI Feedback with Prioritized Layers for a Multi-Carrier System. IEEE Transactions on Wireless Communications 2011, 10(12): 4282-4293

\section{ISSUE DATE:}

2011-12

URL:

http://hdl.handle.net/2433/158644

\section{RIGHT:}

(c) 2011 IEEE. Personal use of this material is permitted. Permission from IEEE must be obtained for all other uses, in any current or future media, including reprinting/republishing this material for advertising or promotional purposes, creating new collective works, for resale or redistribution to servers or lists, or reuse of any copyrighted component of this work in other works.; この論文は出版社版でありません。引用の際には出版社版をご確認ご利用ください。; This is not the published version. Please cite only the published version. 


\title{
Uplink Contention-based CSI Feedback with Prioritized Layers for a Multi-Carrier System
}

\author{
Megumi Kaneko*, Kazunori Hayashi*, Petar Popovski\#, \\ Hiroyuki Yomo $\# \dagger$ and Hideaki Sakai* \\ * Graduate School of Informatics, Kyoto University \\ Yoshida Honmachi Sakyo-ku, Kyoto, 606-8501, Japan \\ \# Department of Electronic Systems, Aalborg University \\ Niels Jernes Vej 12, DK-9220 Aalborg, Denmark \\ $\dagger$ Faculty of Engineering Science, Kansai University, \\ 3-3-35 Yamatecho, Suita, Osaka, 564-8680 Japan \\ Email: \{meg,kazunori,hsakai\}@i.kyoto-u.ac.jp, \{petarp,yomo\}@es.aau.dk
}

\begin{abstract}
Optimized resource allocation of the Downlink (DL) in wireless systems utilizing Multi-Carrier (MC) transmission requires Channel State Information (CSI) feedback for each user/subchannel to the Base Station (BS), consuming a high amount of Uplink (UL) radio resources. To alleviate this problem, several works have considered contention-based CSI feedback in the UL control channel. We propose such a feedback scheme for a generic MC system, based on the idea of variable collision protection, where the probability that a feedback information experiences a collision depends on its importance. By partitioning the CSI into orthogonal layers of priority, and allocating different numbers of feedback slots to each layer, this scheme ensures that the feedback success probability is higher for the CSI with better quality, which is more likely to be used by the scheduler. Furthermore, we present a theoretical performance analysis of the proposed scheme, assuming Maximum CSI (Max CSI) and normalized Proportional Fair Scheduler (PFS), where a tight approximation of the achievable throughput is obtained assuming discrete Adaptive Modulation (AM) and CSI feedback which are relevant for the practical systems. Analytical and simulation results show that our proposed scheme provides an excellent trade-off between system performance and feedback overhead.
\end{abstract}

Index Terms-Radio Resource Allocation, Multi-Carrier (MC) System, Multi-User Diversity, Channel State Information (CSI) Feedback, Proportional Fair Scheduler (PFS)

\section{INTRODUCTION}

The design of radio resource allocation algorithms for Downlink (DL) Multi-Carrier (MC) systems has attracted much attention. MC transmission technology enables to exploit the Multi-User Diversity (MUD) effect [1] with a fine persubchannel granularity for increasing system throughput. To do so, Channel State Information (CSI) knowledge per user and subchannel is required at the Base Station (BS). However, the amount of CSI sent in the Uplink (UL) channel increases linearly with the number of users and subchannels [2]. Such an overhead takes a substantial portion of the total radio resources, directly affecting system throughput [3]. Many works have tackled the issue of CSI feedback reduction assuming sequential feedback per user in the UL control channel, as in [2] where quantized modulation levels are reported subchannelwise or in [4] where feedback parameters are optimized. Further reduction is achieved by [5][6], where only CSIs higher than a predefined threshold are reported, providing MUD gain while reducing the feedback load. In [7][8], a subchannel-wise one-bit feedback scheme is proposed under the assumption of ideal transmission rates. The adaptive feedback encoding method in [9] optimizes the amount of feedback depending on the scheduler's requests. Analysis for the round robin scheduler is provided by [10], where indices of the $M$-best subchannels are reported.

However, the CSI feedback load still increases proportionally to the number of users/subchannels allowed to feed back. A solution pointed out in [5] is to share the UL control channel among users via Random Access (RA) or contention-based feedback, reducing the required UL resource by allowing collisions among CSI packets. Several contention-based CSI feedback protocols have been proposed for a Single Carrier (SC) system by [11][12], the main issue being the optimization of a feedback threshold and access probability. This idea is extended in [13][14][15] where multiple feedback thresholds are optimized. As an SC system is assumed, the common idea is to set the feedback threshold to its highest value at first, then to sequentially lower it, until a user reports. The thresholds are optimized so that only one user with the highest CSI reports. These schemes are extended to the MC case in [16][17], but the feedback load increases proportionally to the number of subchannels, as users contend for each subchannel sequentially. Thus, a specific protocol design is required for an MC system with distributed feedback. A scheme for MC system is proposed by [18], where users contend if all subchannnels in a group exceed a threshold. The performance for infinite number of subchannels is analyzed, but no scheduler is assumed.

In this context, we consider the design of contention-based CSI feedback in the UL control channel, for a generic MC system where the UL CSI overhead consumes useful resources, directly affecting the system throughput. We introduce the key concept of variable collision protection, where the probability that a CSI packet experiences a collision depends on its level of importance. The CSI feedback load is first reduced by only considering the subchannels with good quality, then, depending on the quality level, CSIs are fed back with varying 
collision protection, tuned by the number of slots to feed back each CSI level. As the quality of the CSI reduces, the level of protection diminishes since they have a lower chance to be used by the BS DL scheduler. The main trade-off is between the performance gain provided by variable collision protection and the loss incurred by the feedback of one slot per CSI level, as opposed to the grouped feedback of CSI levels per slot as in the reference scheme based on [5] [11]. The feedback scheme is designed for two schedulers, Maximum CSI (Max CSI) and normalized Proportional Fair Scheduler (PFS). Throughput was analytically derived in [19] for Max CSI. ${ }^{1}$ We generalize this work by improving the protocol and by providing the complete analysis for normalized PFS and deriving the optimal thresholds. Moreover, simulations are performed under realistic fading channels, for which the analysis is still valid, and the utility of the proposed scheme with heterogeneous users is shown.

In addition, most previous works performing throughput analysis assumed the rate to be a strictly increasing function of the continuous Signal-to-Noise Ratio (SNR) $\gamma$ as in [18] [15], instead of a step function, although practical systems utilize discrete Adaptive Modulation (AM) where the rate is quantized. Such a continuous model, which usually determines a unique best rate user to be scheduled, is acceptable for analysis if the achievable rates are continuous [15] or if scheduling is not considered as in [18]. However, it is not applicable when the CSI feedback consists of discrete AM levels, as multiple users may achieve the same best discrete rate and be candidates for scheduling. This is the reason why analysis in [13] for an SC system and in [21] for an MC system are based on the discrete rates. Note that [21] focuses on a sequential CSI feedback scheme and assumes that all users have the same average SNR, which greatly simplifies the analysis. In this work, we derive a tight approximation of the DL throughput given contention-based UL CSI feedback, assuming a discrete AM model and different average user SNRs. Most importantly, the analysis enables us to derive optimal thresholds and feedback slot distribution that maximize the average throughput. Computer simulations attest the improvement of the proposed method over existing ones, as well as confirm the validity of our analysis.

The remainder of the paper is organized as follows. After presenting the system model in Section II, reference schemes and proposed protocols are explained in Section III for two schedulers. These schemes are analyzed in Sections IV, V. Numerical results are presented in Section VI. Finally, conclusions are drawn and directions for future work are given.

\section{System Model}

We focus on DL scheduling in an MC single cell with an UL CSI feedback channel composed of $S$ RA slots, which may be any orthogonal resource such as time, frequency or code, whose required size varies depending on the feedback scheme, hence affecting the system throughput. CSI feedback occurs before scheduling each frame composed of several MC

\footnotetext{
${ }^{1}$ An initial feedback protocol based on random feedback was proposed in [20] but is not considered here.
}

symbols under block-fading. We consider the discrete AM model in Table I, where SNRs are quantized by the SNR thresholds $\sigma_{m}$ with a target Bit Error Rate (BER) of $10^{-6}$ for uncoded Quadrature Amplitude Modulation (QAM) symbols [22]. The discrete instantaneous rate $\nu_{k, n} \in\left\{r_{1}, \ldots, r_{M}\right\}$ of user $k$ among $K$, subchannel $n$ among $N$ with discrete AM level $m=1, \ldots, M$ is given by the SNR threshold immediately below the instantaneous SNR $\gamma_{k, n}{ }^{2}$. The CSI, whose content depends on the feedback scheme (Section III), refers to these discrete rates $\nu_{k, n}$. The full CSI of user $k$ is the set of $N$ discrete reported rates $\nu_{k, n}, n=1, \ldots, N$. The discrete AM model implies that many users may have the same achievable rate $\nu_{k, n}=r_{m}$, if their $\gamma_{k, n}$ fall into the same SNR region. This effect is not captured by the continuous rate model which is strictly increasing with respect to $\gamma_{k, n}$ (Section I). Thus, it is essential to consider the discrete user rate statistics for deriving the analytical throughput. One might argue that the continuous rate model should be used to account for BER that partly smoothes the achieved throughput. Although the achieved throughput is degraded by bit errors, Section VI shows that our analysis with discrete rates closely approaches the simulation results with bit error degradation. This confirms the necessity of considering the discrete rate model in the analysis, while BER effects may be neglected.

TABLE I

Discrete Adaptive Modulation Model

\begin{tabular}{|l|c|c|c|c|c|}
\hline Modulation & BPSK & QPSK & 16-QAM & 64-QAM & 256-QAM \\
\hline AM Level $m$ & 1 & 2 & 3 & 4 & 5 \\
\hline $\begin{array}{l}\text { Rate } r_{m}\left(\bar{r}_{m}\right) \\
{[\mathrm{b} / \text { symbol] }}\end{array}$ & 1 & 2 & 4 & 6 & 8 \\
\hline $\begin{array}{l}\text { SNR Threshold } \\
\sigma_{m}[\mathrm{~dB}]\end{array}$ & -5 & 13.6 & 20.6 & 26.8 & 32.9 \\
\hline
\end{tabular}

The discrete average user rate $\bar{\nu}_{k} \in\left\{r_{1}, \ldots, r_{M}\right\}$ of user $k$ is given by the SNR threshold immediately below the longterm average user SNR $\bar{\gamma}_{k}$ which depends solely on the user distance to BS. Note that $\bar{\gamma}_{k}$, hence $\bar{\nu}_{k}$, varies across users. $\bar{\nu}_{k}$ can be assumed known at the BS, as it is slowly varying and needs to be updated seldomly. If no instantaneous CSI is available at the BS for a subchannel due to absence of feedback, a random user is scheduled. ${ }^{3}$

\section{Conventional And Proposed UL CSI FEedBack SCHEMES}

\section{A. Conventional Schemes}

We adopt the following collision model: if two users or more select the same slot for feedback, collision occurs and the CSI for all the involved users is lost. Packet errors due to channel fading and noise are not considered in the UL feedback channel as in [11] [12], i.e., a high level of protection is provided thanks to low modulation levels and/or coding. In the reference Full CSI-RA (FRA) scheme, all users try to feed back their full CSI. Given $S$ slots for RA, each user selects one

\footnotetext{
${ }^{2}$ The proposed feedback scheme can work with any other discrete AM model and larger sets, including coding.

${ }^{3}$ It is also possible to schedule the user with the best average rate in Max CSI, and derive its analytical throughput.
} 
slot among $S$. If user $k$ picks slot $s$, his full CSI is successfully transmitted to the BS if no other user selects the same slot. If several users select the same slot, all the CSI for those users is lost. A user CSI, sent in one slot $s$, is composed of the user ID, the AM level per subchannel, and Cyclic Redundancy Check (CRC) bits. As $S$ slots are reserved in the feedback channel of the system, the total number of bits $B_{\mathrm{FRA}}$ to be reserved for feedback is

$$
B_{\mathrm{FRA}}=S \times\left(b_{\mathrm{ID}}+\left\lceil\log _{2} M\right\rceil \times N+b_{\mathrm{CRC}}\right),
$$

where $b_{\text {ID }}$ denotes the number of bits used for user ID, $\log _{2} M$ the number of bits required for encoding the $M$ AM levels, $\lceil$.$\rceil the ceil function, and b_{\mathrm{CRC}}$ the number of bits for CRC.

In the second reference scheme, Threshold-RA (TRA), only users with subchannel SNRs higher than a certain threshold, say AM level 4, feed back, or equivalently the $L$-best AM levels, $L=1, \ldots, M$. For $L=2$, a user having subchannels with level 4 or 5 feeds back using one slot among $S$. This is an extension of the threshold based schemes in [5][11] for $\mathrm{SC}$ system, where a user feeds back if his channel is above a certain threshold. Each user who feeds back needs to specify if each subchannel has AM level 5, 4, or below. The total number of bits $B_{\text {TRA }}$ is

$$
B_{\mathrm{TRA}}=S \times\left(b_{\mathrm{ID}}+\left\lceil\log _{2}(L+1)\right\rceil \times N+b_{\mathrm{CRC}}\right) .
$$

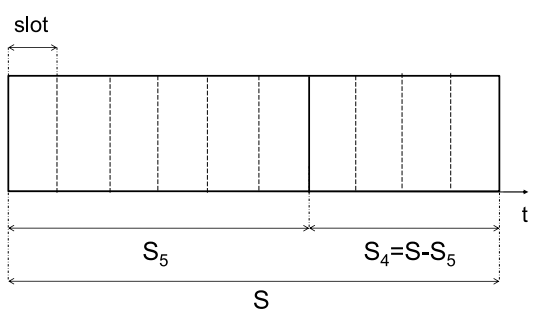

Fig. 1. Proposed RA channel, example with $S=10, L=2$.

\section{B. Proposed OMax Scheme For Max CSI}

The Max CSI algorithm allocates in each subchannel the user with the highest instantaneous SNR $\gamma_{k, n}$. In the proposed Orthogonal-RA for Max CSI (OMax) scheme, users feed back the CSIs of the subchannels that support the $L$-absolute best AM levels. For example, with $L=2$, there are two levels of priority, the best level composed of all the subchannels of all users with AM level $5, \nu_{k, n}=r_{5}$; and the second best level for AM level $4, \nu_{k, n}=r_{4}$. As illustrated in Fig. 1, the $S$ RA slots are partitioned given these distinct levels: $S_{5}$ slots for reporting AM level 5 and $S_{4}$ for level 4. As it will be shown in the analysis in Section IV, the slot distribution that maximizes the average throughput is computed at the BS and notified to the Mobile Stations (MSs) every time the optimal slot distribution changes, which is not often. A user having one or more subchannels with level 5 selects one slot among $S_{5}$ and reports them in that slot, with the encoding method specified below, and similarly for level 4 . A user having both subchannels with level 5 and 4 will use two slots, one among $S_{5}$ and another among $S_{4}$. Thus, compared to TRA, OMax may have a higher collision probability for a given requested number of levels $L$, as one slot is chosen per level versus one slot per user having subchannels above the threshold. However, OMax provides a variable collision protection, where higher quality CSI experiences less collisions compared to lower quality CSI, as subchannels with higher AM levels have a higher probability to be scheduled, and the analysis will determine the optimal partition of $S_{5}$ and $S_{4}$ that maximizes the overall throughput. The user CSI is composed of the user ID, one AM level and CRC bits. As one AM level is coded per slot, there is one bit per subchannel indicating if this level is supported or not. The AM level is first coded, followed by $N$ bits with 0 or 1 (in conventional schemes, the AM level is coded per subchannel). The total number of feedback bits $B_{\text {OMax }}{ }^{4}$ is

$$
B_{\mathrm{OMax}}=S \times\left(b_{\mathrm{ID}}+\left\lceil\log _{2} M\right\rceil+N+b_{\mathrm{CRC}}\right) .
$$

\section{Proposed OPFS Scheme For PFS}

Although the above scheme may ensure a high MUD gain, it may decrease fairness as users with lower channel qualities would not be scheduled. To counter this, we introduce the feedback of relative-best levels, referred as Orthogonal-RA for PFS (OPFS), for the normalized PFS algorithm in [13][15], where subchannel $n$ is allocated to the user with the best ratio $\rho_{k, n}=\frac{\nu_{k, n}}{\bar{\nu}_{k}}$. Thus, a user is allocated when his instantaneous channel state is high compared to his average channel quality. As defined in Section II, both instantaneous rate $\nu_{k, n}$ and average rate $\bar{\nu}_{k}$ take the discrete and finite values in Table I, so the ratio $\rho_{k, n}$ is also discrete and finite, as shown in Table II. The set of ratio values is denoted $\mathcal{Q}$, where the elements are ordered in increasing order, and $Q=\operatorname{card}(\mathcal{Q})$. $\rho_{k, n} \in\left\{\rho_{1}, \ldots, \rho_{Q}\right\}$ can take these $Q$ different values. A user reports his $L$-relative best levels, enabling users with lower levels to be scheduled. As all users would feed back, increasing collisions, we define additional thresholds that set the levels of priority for the channel ratios. If we set thresholds $(\alpha, \beta) \in \mathcal{Q}^{2}$ such that $\beta<\alpha$, there are 2 regions of level for feedback, i.e., region $\mathbf{S}_{\alpha}$ with $S_{\alpha}$ slots used for $\rho_{k, n} \in\left[\alpha, \infty\left[\right.\right.$ and region $\mathbf{S}_{\beta}$ with $S_{\beta}$ slots for $\rho_{k, n} \in\left[\beta, \alpha\right.$, and $S_{\alpha}+S_{\beta}=S$. In Fig. $1, S_{\alpha}$ corresponds to $S_{5}$ and $S_{\beta}$ to $S_{4}$. If $L=2$ relative best levels are requested, each user identifies his subchannels with the 2 -relative best ratios. Let us note $\lambda_{k, 1}=\left\{n \mid \rho_{k, n}=\rho_{k, 1 \mathrm{rb}}\right\}$, the set of subchannels of user $k$ whose ratios are equal to the relative best ratio of level $\rho_{k, 1 \mathrm{rb}}$. Likewise, $\lambda_{k, 2}=\left\{n \mid \rho_{k, n}=\right.$ $\left.\rho_{k, 2 \mathrm{rb}}\right\}$ denotes the set of subchannels whose ratios are equal to the second best level $\rho_{k, 2 \mathrm{rb}}$. Then, if $\rho_{k, 1 \mathrm{rb}} \in[\beta, \alpha[$, user $k$ selects one slot among $S_{\beta}$; if $\rho_{k, 1 \mathrm{rb}} \in[\alpha, \infty[$, he selects one slot among $S_{\alpha}$. Otherwise, there is no feedback. The same applies to $\rho_{k, 2 \mathrm{rb}}$. Simply, only one slot will be used in 2 cases: either $\rho_{k, 1 \mathrm{rb}} \in\left[\alpha, \infty\left[\right.\right.$ and $\left.\rho_{k, 2 \mathrm{rb}} \in\right]-\infty, \beta\left[\right.$, or $\rho_{k, 1 \mathrm{rb}} \in[\beta, \alpha[$ and $\left.\rho_{k, 2 \mathrm{rb}} \in\right]-\infty, \beta[$. Two slots will be used in 3 cases: first, if both $\rho_{k, 1 \mathrm{rb}}$ and $\rho_{k, 2 \mathrm{rb}} \in\left[\beta, \alpha\left[, 2\right.\right.$ slots are chosen in $\mathbf{S}_{\beta}$, second, if both are in $\left[\alpha, \infty\left[, 2\right.\right.$ slots are chosen in $\mathbf{S}_{\alpha}$, or

\footnotetext{
${ }^{4}$ We can reduce $B_{\text {OMax }}$ by removing $\left\lceil\log _{2} M\right\rceil$, as BS may guess the AM level from slot $s$, either in $S_{4}$ or $S_{5}$. For OPFS, up to $\left\lceil\log _{2} M\right\rceil$ bits will be required. Thus, $B_{\mathrm{OMax}}$ gives an upper bound on the number of feedback bits of $O M a x$ and $O P F S$.
} 
finally, if $\rho_{k, 1 \mathrm{rb}} \in\left[\alpha, \infty\left[\right.\right.$ and $\rho_{k, 2 \mathrm{rb}} \in[\beta, \alpha[$, then one slot is taken from each region. Again, one slot is chosen per ratio value, i.e., per set $\lambda_{k, 1}$ or $\lambda_{k, 2}$, and corresponds to one AM level as the user's average rate is constant over subchannels and known at the BS, requiring $B_{\text {OMax }}$ feedback bits as OMax. In the reference TRA scheme for PFS, users with subchannels whose ratios are above the basic threshold $\beta$ are allowed to feed back. In one slot, all the AM levels of these subchannels are encoded. As a subchannel may take any AM level, the number of feedback bits is equal to $B_{\mathrm{FRA}}$.

TABLE II

RATIO VALUES TAKEN BY $\rho_{k, n}=\frac{\nu_{k, n}}{\bar{\nu}_{k}}$ FOR ANY USER $k$, SUBCHANNEL $n$, wITH $\nu_{k, n}=r_{m}, \bar{\nu}_{k}=\bar{r}_{j}$ AND $m, j=1, \ldots, M$.

\begin{tabular}{|l|c|c|c|c|c|}
\hline$\rho_{q}, q \in[1 . . Q]$ & $\bar{r}_{1}$ & $\bar{r}_{2}$ & $\bar{r}_{3}$ & $\bar{r}_{4}$ & $\bar{r}_{5}$ \\
\hline$r_{1}$ & 1 & $1 / 2$ & $1 / 4$ & $1 / 6$ & $1 / 8$ \\
\hline$r_{2}$ & 2 & 1 & $1 / 2$ & $1 / 3$ & $1 / 4$ \\
\hline$r_{3}$ & 4 & 2 & 1 & $2 / 3$ & $1 / 2$ \\
\hline$r_{4}$ & 6 & 3 & $3 / 2$ & 1 & $3 / 4$ \\
\hline$r_{5}$ & 8 & 4 & 2 & $4 / 3$ & 1 \\
\hline
\end{tabular}

\section{Performance Analysis for Max CSI Algorithm}

In the analysis, throughput is determined for a subchannel, as it is defined as the sum of per-subchannel throughput divided by the total number of subchannels $N$, which have independent instantaneous SNRs. The whole analysis is MC specific as the successful report of the CSI for a subchannel depends on the channel occurrences of the other users on the other subchannels. We consider a simplified cell model, i.e., a circular cell of radius $R$ where users are uniformly generated. The distance of a user $k$ 's location to the cell center (BS) is denoted $x_{k}$. The joint probability distribution of $\gamma_{k, n}$ and $x_{k}$ is $p\left(\gamma_{k, n}, x_{k}\right)=p\left(\gamma_{k, n} \mid x_{k}\right) p\left(x_{k}\right)$, where $p\left(\gamma_{k, n} \mid x_{k}\right)$ is the conditional probability of the instantaneous SNR given the user location and $p\left(x_{k}\right)$ is the probability to have this user location. Assuming Rayleigh fading environments, the instantaneous SNR $\gamma_{k, n}$ follows an exponential distribution, $p\left(\gamma_{k, n} \mid x_{k}\right)=\frac{1}{\bar{\gamma}_{k}} e^{-\frac{\gamma_{k}, n}{\bar{\gamma}_{k}}}$, where $\bar{\gamma}_{k}$ denotes the average SNR of this user ${ }^{5}$. Fixing the average SNR to be $0 \mathrm{~dB}$ at the edge of the cell ${ }^{6}$, we have $\bar{\gamma}_{k}=\left(\frac{R}{x_{k}}\right)^{\alpha_{\exp }}$, where $\alpha_{\exp }$ is the path loss exponent chosen equal to 3 . Under the assumption of uniform user distribution, we obtain $p\left(x_{k}\right)=\frac{2 x_{k}}{R^{2}}$.

\section{A. Analysis for the Proposed CSI Feedback Scheme OMax}

We determine the probability that the best AM level $r_{5}$ is allocated on a certain subchannel $n$. In the sequel, subscript $n$ is omitted for the expressions that are the same for any subchannel $n=1, \ldots, N$. For a user $k$ among $K$ users in the cell, let $P_{\mathrm{OM}, \mathrm{SR}, k}\left(r_{5} \mid x_{1}, \ldots, x_{K}\right)$ be the joint conditional probability of supporting $r_{5}$ on subchannel $n$ and of reporting successfully for given $x_{1}, \ldots, x_{K}$. We define $P\left(\nu_{k, n}=r_{m} \mid x_{k}\right)$, hereafter denoted $P\left(r_{m} \mid x_{k}\right)$, the conditional probability mass

\footnotetext{
${ }^{5}$ The shadowing effect is not included as it renders the analysis too complex. However, it may be considered in future work.

${ }^{6}$ To generalize by fixing the cell edge SNR level at $\sigma_{e}$, we can define $\bar{\gamma}_{k}=\sigma_{e}\left(\frac{R}{x_{k}}\right)^{\alpha \exp }$ and the analysis holds.
}

function (pmf) for a given user position $x_{k}$ that subchannel $n$ supports $r_{m}$

$$
P\left(r_{m} \mid x_{k}\right)=\int_{\sigma_{m}}^{\sigma_{m+1}} \frac{1}{\overline{\gamma_{k}}} e^{-\frac{\gamma}{\bar{\gamma}_{k}}} d \gamma=e^{-\frac{\sigma_{m}}{\bar{\gamma}_{k}}}-e^{-\frac{\sigma_{m+1}}{\bar{\gamma}_{k}}},
$$

with $\sigma_{m}$ expressed in linear and $\sigma_{M+1}=+\infty$. $G_{5, x_{i}}=1-$ $\left(1-P\left(r_{5} \mid x_{i}\right)\right)^{N}$ is the probability that at least one subchannel among $N$ supports $r_{5}$ for user $i$. There is successful report if there is no collision with the other $K-1$ users having at least one subchannel among $N$ with $r_{5}$, so

$$
P_{\mathrm{OM}, \mathrm{SR}, k}\left(r_{5} \mid x_{1}, \ldots, x_{K}\right)=P\left(r_{5} \mid x_{k}\right) \prod_{i=1, i \neq k}^{K}\left(1-\frac{G_{5, x_{i}}}{S_{5}}\right),
$$

as there are $S_{5}$ slots for this layer. As users have different positions $x_{1}, \ldots, x_{K}$ which are independent random variables, we get after marginalization with respect to all positions $x_{i} \in$ ] $0, R]$,

$$
\begin{aligned}
P_{\mathrm{OM}, \mathrm{SR}, k}\left(r_{5}\right) & =\int_{x_{1}=0}^{R} \cdots \int_{x_{K}=0}^{R} P_{\mathrm{OM}, \mathrm{SR}, k}\left(r_{5} \mid x_{1}, \ldots, x_{K}\right) \\
& \times p\left(x_{1}\right) \cdots p\left(x_{K}\right) d x_{1} \cdots d x_{K}
\end{aligned}
$$

and $P_{\mathrm{OM}, \mathrm{SR}, k}\left(r_{5}\right)=I_{1} \times \cdots \times I_{k} \times \cdots \times I_{K}$, where

$$
\begin{aligned}
I_{i} & =\int_{x_{i}=0}^{R}\left(1-\frac{G_{5, x_{i}}}{S_{5}}\right) \frac{2 x_{i}}{R^{2}} d x_{i} \text { for } i \neq k, \\
I_{k} & =\int_{x_{k}=0}^{R} P\left(r_{5} \mid x_{k}\right) \frac{2 x_{k}}{R^{2}} d x_{k} .
\end{aligned}
$$

First, let us determine $I_{k}=\int_{x_{k}=0}^{R} \frac{2 x_{k}}{R^{2}} e^{-\frac{\sigma_{5} x_{k}^{3}}{R^{3}}} d x_{k}$. With a change of variables $y=x_{k}^{3}$, we write $I_{k}=$ $\int_{y=0}^{R^{3}} \frac{2}{3 R^{2}} y^{-1 / 3} e^{-\frac{\sigma_{5} y}{R^{3}}} d y$. Using (3.381-1) in [23] we can show that

$$
I_{k}=\frac{2}{3}\left(\sigma_{5}\right)^{-2 / 3} \Gamma(2 / 3) \gamma_{\text {inc }}\left(\sigma_{5}, 2 / 3\right) \equiv P\left(r_{5}\right),
$$

where $\Gamma$ denotes the gamma function defined as $\Gamma(z)=$ $\int_{0}^{\infty} e^{-t} t^{z-1} d t$, and $\gamma_{\text {inc }}$ the incomplete gamma function, defined as $\gamma_{\text {inc }}(z, u)=\int_{0}^{u} e^{-t} t^{z-1} d t$ [23]. Next, we calculate $I_{i}$, which can be written $I_{i}=1-\frac{G_{5}}{S_{5}}$, where, using Newton's binomial theorem,

$$
\begin{aligned}
G_{5} & =1-\int_{x_{i}=0}^{R} \frac{2 x_{i}}{R^{2}}\left(1-e^{-\frac{\sigma_{5} x_{i}^{3}}{R^{3}}}\right)^{N} d x_{i} \\
& =1-\int_{y=0}^{R^{3}} \frac{2}{3 R^{2}} y^{-1 / 3} \sum_{a=0}^{N} \mathrm{C}_{N}^{a}(-1)^{a} e^{-\frac{\sigma_{5} a y}{R^{3}}} d y,
\end{aligned}
$$

with the change of variables $y=x_{i}^{3}$ and $\mathrm{C}_{N}^{a}=\frac{N !}{a !(N-a) !}$. Using (3.381-1) in [23], we get

$G_{5}=1-\sum_{a=0}^{N} \mathrm{C}_{N}^{a}(-1)^{a} \frac{2}{3}\left(\sigma_{5} a\right)^{-2 / 3} \Gamma(2 / 3) \gamma_{\mathrm{inc}}\left(\sigma_{5} a, 2 / 3\right)$.

The joint probability of supporting $r_{5}$ and successful report for a certain user is given by

$$
P_{\mathrm{OM}, \mathrm{SR}}\left(r_{5}\right)=P_{\mathrm{OM}, \mathrm{SR}, k}\left(r_{5}\right)=P\left(r_{5}\right)\left(1-\frac{G_{5}}{S_{5}}\right)^{K-1},
$$


where index $k$ is dropped as users are generated uniformly in the cell. Similarly, we get the joint probability of having $r_{4}$ on subchannel $n$ and of successful report $P_{\mathrm{OM}, \mathrm{SR}}\left(r_{4}\right)$. Here a user with $r_{4}$ competes with $K-1$ competitors who have at least one subchannel with $r_{4}$ among $N$. Thus,

$$
P_{\mathrm{OM}, \mathrm{SR}}\left(r_{4}\right)=P\left(r_{4}\right)\left(1-\frac{G_{4}}{S-S_{5}}\right)^{K-1},
$$

with $S_{5}<S, P\left(r_{4}\right)=\frac{2}{3}\left(\sigma_{4}\right)^{-2 / 3} \Gamma(2 / 3) \gamma_{\text {inc }}\left(\sigma_{4}, 2 / 3\right)-$ $P\left(r_{5}\right)$ and

$$
\begin{aligned}
G_{4} & =1-\sum_{a=0}^{N} \mathrm{C}_{N}^{a}(-1)^{a} \sum_{b=0}^{a} \mathrm{C}_{a}^{b}(-1)^{a-b} \Gamma\left(\frac{2}{3}\right) \\
& \times \frac{2}{3}\left(\sigma_{5} b+\sigma_{4}(a-b)\right)^{-\frac{2}{3}} \gamma_{\mathrm{inc}}\left(\sigma_{5} b+\sigma_{4}(a-b), \frac{2}{3}\right) .
\end{aligned}
$$

Denoting $P_{\mathrm{OM}}\left(r_{i}\right)$ the probability to allocate rate $r_{i}, i=4,5$, the throughput is approximated by

$$
\tau_{\mathrm{OM}}=r_{5} P_{\mathrm{OM}}\left(r_{5}\right)+r_{4} P_{\mathrm{OM}}\left(r_{4}\right)+r_{\mathrm{rnd}} P_{\mathrm{OM}, \text { out }},
$$

where $P_{\mathrm{OM}}\left(r_{5}\right)=1-\left(1-P_{\mathrm{OM}, \mathrm{SR}}\left(r_{5}\right)\right)^{K}$ expresses that at least one user with $r_{5}$ has successful report and $P_{\mathrm{OM}}\left(r_{4}\right)=$ $\left(1-P_{\mathrm{OM}, \mathrm{SR}}\left(r_{5}\right)\right)^{K}-\left(1-P_{\mathrm{OM}, \mathrm{SR}}\left(r_{5}\right)-P_{\mathrm{OM}, \mathrm{SR}}\left(r_{4}\right)\right)^{K}$ expresses that at least one user with $r_{4}$ has successful report and everybody with $r_{5}$ failed. Actually, $P_{\mathrm{OM}}\left(r_{i}\right)$ are approximate expressions as they implicitly assume that the probabilities of no collisions among users are independent. However, finding the exact probability becomes rapidly intractable as $S$ and/or $K$ grow, as one needs to count all the possible patterns of users' choices of slots and to retain the ones where a slot was chosen by a unique user (variables $k_{1}, \ldots, k_{S}$ representing the number of users that picked each slot follow the multinomial distribution). We have verified numerically that the approximation closely matches the exact expression as $K$ increases, but we cannot present the verification here due to lack of space. In addition, the simulation results in Section VI will corroborate this approximation.

There is outage in the absence of feedback. To determine the outage rate, we can write $P_{\mathrm{OM} \text {,out }}=1-P_{\mathrm{OM}}\left(r_{5}\right)-P_{\mathrm{OM}}\left(r_{4}\right)$, and $r_{\text {rnd }}=\sum_{m=1}^{M} r_{m} P\left(\sigma_{m} \leq \bar{\gamma}_{\text {rnd }}<\sigma_{m+1}\right)$, i.e.,

$$
\begin{aligned}
r_{\mathrm{rnd}} & =r_{1}\left[1-\left(\frac{1}{\sigma_{2}}\right)^{\frac{2}{3}}\right]+r_{M}\left(\frac{1}{\sigma_{L}}\right)^{\frac{2}{3}} \\
& +\sum_{m=2}^{M-1} r_{m}\left[\left(\frac{1}{\sigma_{m}}\right)^{\frac{2}{3}}-\left(\frac{1}{\sigma_{m+1}}\right)^{\frac{2}{3}}\right],
\end{aligned}
$$

as a random user is allocated the rate given by his average SNR level $\bar{\gamma}_{\text {rnd }} \cdot{ }^{7}$ In (13), the effects of decoding errors are not considered unlike in the simulations conducted in Section VI. Note that, although it is hard to guess the behavior of the throughput by merely looking at the analytical formula (which is only expected, given the complexity of the system and number of parameters), this expression enables to obtain the throughput behavior against multiple parameters at once, as well as parameter optimization, which would have been

\footnotetext{
${ }^{7}$ Eq. (14) assumes that the user distribution is uniform even in case of outage, but matches well the exact outage.
}

hardly possible with simulations only. Parameter optimization is discussed in detail in Section VI.

\section{B. Analysis for Full CSI RA Feedback}

Now every user feeds back and the best rate user is scheduled on subchannel $n$. Denoting $P_{\mathrm{FM}}\left(r_{m}\right)$ the probability to allocate $r_{m}, m=1, \ldots, M$, the throughput is approximated by

$$
\tau_{\mathrm{FM}}=\sum_{m=1}^{M} r_{m} P_{\mathrm{FM}}\left(r_{m}\right)+r_{\mathrm{rnd}} P_{\mathrm{FM}, \mathrm{out}},
$$

where, defining $P_{\mathrm{FM}, \mathrm{SR}}\left(r_{m}\right)=P\left(r_{m}\right)\left(1-\frac{1}{S}\right)^{K-1}$, we have $P_{\mathrm{FM}}\left(r_{5}\right)=1-\left(1-P_{\mathrm{FM}, \mathrm{SR}}\left(r_{5}\right)\right)^{K}$ and, for $m<$ $M, P_{\mathrm{FM}}\left(r_{m}\right)=\left(1-\sum_{i=m+1}^{M} P_{\mathrm{FM}, \mathrm{SR}}\left(r_{i}\right)\right)^{K}-(1-$ $\left.\sum_{i=m+1}^{M} P_{\mathrm{FM}, \mathrm{SR}}\left(r_{i}\right)-P_{\mathrm{FM}, \mathrm{SR}}\left(r_{m}\right)\right)^{K}$ expresses that at least one user with $r_{m}$ has successful report and everybody with higher rates failed. Moreover, $P_{\mathrm{FM} \text {,out }}=1-\sum_{m=1}^{M} P_{\mathrm{FM}}\left(r_{m}\right)$.

\section{Analysis for Threshold CSI RA Feedback}

In this scheme, a user feeds back if at least one of his subchannels supports $r_{5}$ or $r_{4}$, expressed by probability $G_{4,5}=$ $1-\sum_{a=0}^{N} \mathrm{C}_{N}^{a}(-1)^{a} \frac{2}{3}\left(\sigma_{4} a\right)^{-2 / 3} \Gamma(2 / 3) \gamma_{\text {inc }}\left(\sigma_{4} a, 2 / 3\right)$. Denoting $P_{\mathrm{TM}}\left(r_{i}\right)$ the probability to allocate $r_{i}, i=4,5$, the throughput is approximated by

$$
\tau_{\mathrm{TM}}=r_{5} P_{\mathrm{TM}}\left(r_{5}\right)+r_{4} P_{\mathrm{TM}}\left(r_{4}\right)+r_{\mathrm{rnd}} P_{\mathrm{TM}, \text { out }},
$$

where $P_{\mathrm{TM}}\left(r_{5}\right)=1-\left(1-P_{\mathrm{TM}, \mathrm{SR}}\left(r_{5}\right)\right)^{K}, P_{\mathrm{TM}}\left(r_{4}\right)=$ $\left(1-P_{\mathrm{TM}, \mathrm{SR}}\left(r_{5}\right)\right)^{K}-\left(1-P_{\mathrm{TM}, \mathrm{SR}}\left(r_{5}\right)-P_{\mathrm{TM}, \mathrm{SR}}\left(r_{4}\right)\right)^{K}$ and $P_{\mathrm{TM}, \mathrm{SR}}\left(r_{i}\right)=P\left(r_{i}\right)\left(1-\frac{G_{4,5}}{S}\right)^{K-1}$.

\section{Performance Analysis for PFS Algorithm}

\section{A. Analysis for the Proposed CSI Feedback Scheme OPFS}

We determine the achievable throughput of the OPFS scheme. The analysis becomes more involved as the scheduling decision is based on the user ratios (instantaneous rate over average rate), while the throughput is determined by the actual rate. Again, we consider the allocation on a subchannel $n$ and omit subscript $n$. If several users successfully report the same value of the best ratio $\rho$, the one having the best rate $r$ is selected for scheduling. This assumption, in addition to simplifying the analysis, offers a practical way to increase the throughput while maintaining the same degree of proportional fairness. Let us focus on the region $\mathbf{S}_{\alpha}$ where only subchannels with ratios $\rho \geq \alpha$ are fed back. A user successfully reports a channel occurrence $\left(\rho_{k, n}, \nu_{k, n}\right)=\left(\rho_{q}, r_{m}\right)$ if $\rho_{q}$ belongs either to the best or second best level among his $N$ subchannels, and there is no collision with any of the other $K-1$ competitors. Denoting $P_{\mathrm{OP}, \alpha, \mathrm{SR}, k}\left(\rho_{q}, r_{m}\right)$ the probability that user $k$ successfully reports $\left(\rho_{q}, r_{m}\right)$ for subchannel $n$, we have

$$
P_{\mathrm{OP}, \alpha, \mathrm{SR}, k}\left(\rho_{q}, r_{m}\right)=F_{\mathrm{OP}, \alpha, k}\left(\rho_{q}, r_{m}\right) \prod_{k^{\prime}=1, k^{\prime} \neq k}^{K} H_{\mathrm{OP}, \alpha, k^{\prime}},
$$

with $F_{\mathrm{OP}, \alpha, k}\left(\rho_{q}, r_{m}\right)$ the probability that $\left(\rho_{q}, r_{m}\right)$ is fed back by user $k$, i.e., $\rho_{q}$ belongs either to the best or second best 
level, and $H_{\mathrm{OP}, \alpha, k^{\prime}}$, the probability of having no collision with user $k^{\prime}$.

As users are uniformly generated, index $k$ may be dropped in $F_{\mathrm{OP}, \alpha, k}\left(\rho_{q}, r_{m}\right)$. To calculate $F_{\mathrm{OP}, \alpha}\left(\rho_{q}, r_{m}\right)$, we have to determine all the cases where the considered subchannel $n$ has the best level $\left(\rho_{q}, r_{m}\right)$ among $N$ subchannels, denoted $F_{\mathrm{OP}, \alpha, 1 \mathrm{~b}}\left(\rho_{q}, r_{m}\right)$, or the second best, denoted $F_{\mathrm{OP}, \alpha, 2 \mathrm{~b}}\left(\rho_{q}, r_{m}\right)$, then $F_{\mathrm{OP}, \alpha}\left(\rho_{q}, r_{m}\right)=F_{\mathrm{OP}, \alpha, 1 \mathrm{~b}}\left(\rho_{q}, r_{m}\right)+$ $F_{\mathrm{OP}, \alpha, 2 \mathrm{~b}}\left(\rho_{q}, r_{m}\right)$. For $\left(\rho_{k, n}, \nu_{k, n}\right)=\left(\rho_{q}, r_{m}\right)$, there exists a unique $j=1, \ldots, M$ such that $\bar{\nu}_{k}=\frac{r_{m}}{\rho_{q}} \stackrel{\text { def }}{=} \bar{r}_{j}$. Although $r_{m}$ and $\bar{r}_{j}$ take values in the same set, we use this notation to avoid any confusion. $\bar{r}_{j}$ being constant over user $k$ 's $N$ subchannels, the fact that $n$ has the best ratio $\rho_{q}$ is equivalent to having the best rate $r_{m}$. As subchannels are i.i.d. given $\bar{\gamma}$, the probability that $r_{m}$ is the best among all subchannels is equal to the product of the probability conditioned over $\bar{\gamma}$ that subchannel $n$ has $r_{m}$ and the $N-1$ others have $r_{i} \leq r_{m}$. Then, integrating over the SNR region that gives $\bar{r}_{j}$ and for $\rho_{q} \geq \alpha$,

$$
\begin{aligned}
F_{\mathrm{OP}, \alpha, 1 \mathrm{~b}}\left(\rho_{q}, r_{m}\right) & =\int_{\frac{R}{\sigma_{j+1}^{1 / 3}}}^{\frac{R}{\sigma_{j}^{1 / 3}}} P\left(r_{m} \mid x_{k}\right)\left(\sum_{i=1}^{m} P\left(r_{i} \mid x_{k}\right)\right)^{N-1} \\
& \times p\left(x_{k}\right) d x_{k}
\end{aligned}
$$

with $P\left(r_{m} \mid x_{k}\right)$ the probability to achieve $r_{m}$ given user position $x_{k}$, whose range corresponds to $\bar{\gamma} \in\left[\sigma_{j}, \sigma_{j+1}[\right.$. With the change of variables $y=x_{k}^{3}$, we have

$$
\begin{aligned}
& F_{\mathrm{OP}, \alpha, 1 \mathrm{~b}}\left(\rho_{q}, r_{m}\right)=\int_{\frac{R^{3}}{\sigma_{j+1}}}^{\frac{R^{3}}{\sigma_{j}}}\left[\int_{\sigma_{m}}^{\sigma_{m+1}} \frac{y}{R^{3}} e^{-\frac{\gamma y}{R^{3}}} d \gamma\right] \\
& \times\left[\int_{1}^{\sigma_{m+1}} \frac{y}{R^{3}} e^{-\frac{\gamma y}{R^{3}}} d \gamma\right]^{N-1} \frac{2}{3 R^{2}} y^{-\frac{1}{3}} d y .
\end{aligned}
$$

After some calculations, we obtain $F_{\mathrm{OP}, \alpha, 1 \mathrm{~b}}\left(\rho_{q}, r_{m}\right)=I+$ $I_{0}-J-J_{0}$ where

$$
\begin{aligned}
I= & \sum_{n=1}^{N-1}(-1)^{n} \frac{2}{3} \Gamma\left(\frac{2}{3}\right)\left(\sigma_{m}+n \sigma_{m+1}\right)^{-\frac{2}{3}} \times \\
J & {\left[\gamma_{\mathrm{inc}}\left(\frac{\sigma_{m}+n \sigma_{m+1}}{\sigma_{j}}, \frac{2}{3}\right)-\gamma_{\mathrm{inc}}\left(\frac{\sigma_{m}+n \sigma_{m+1}}{\sigma_{j+1}}, \frac{2}{3}\right)\right], } \\
& \sum_{n=1}^{N-1}(-1)^{n} \frac{2}{3} \Gamma\left(\frac{2}{3}\right)\left((n+1) \sigma_{m+1}\right)^{-\frac{2}{3}} \times \\
I_{0}= & \frac{2}{3} \Gamma\left(\frac{2}{3}\right)\left(\sigma_{m}\right)^{-\frac{2}{3}}\left[\gamma_{\mathrm{inc}}\left(\frac{\sigma_{m}}{\sigma_{j}}, \frac{2}{3}\right)-\gamma_{\mathrm{inc}}\left(\frac{\sigma_{m}}{\sigma_{j+1}}, \frac{2}{3}\right)\right], \\
J_{0} & \left.\left.=\frac{2}{3} \Gamma\left(\frac{2}{3}\right)\left(\sigma_{m+1}\right)^{-\frac{2}{3}}, \frac{2}{3}\right)-\gamma_{\mathrm{inc}}\left(\frac{(n+1) \sigma_{m+1}}{\sigma_{j+1}}, \frac{2}{3}\right)\right], \\
& \times\left[\gamma_{\mathrm{inc}}\left(\frac{\sigma_{m+1}}{\sigma_{j}}, \frac{2}{3}\right)-\gamma_{\mathrm{inc}}\left(\frac{\sigma_{m+1}}{\sigma_{j+1}}, \frac{2}{3}\right)\right] .
\end{aligned}
$$

Similarly, noting $r_{a}$ the rate of the best level, the probability that $r_{m}$ is second best is

$$
\begin{aligned}
& F_{\mathrm{OP}, \alpha, 2 \mathrm{~b}}\left(\rho_{q}, r_{m}\right)=\sum_{a=m+1}^{M} \int_{x_{k}=\frac{R}{\sigma_{j+1}^{1 / 3}}}^{\frac{R}{\sigma_{j}^{1 / 3}}} P\left(r_{m} \mid x_{k}\right) P\left(r_{a} \mid x_{k}\right) \\
& \times\left[P\left(r_{a} \mid x_{k}\right)+\sum_{i=1}^{m} P\left(r_{i} \mid x_{k}\right)\right]^{N-2} p\left(x_{k}\right) d x_{k} .
\end{aligned}
$$

Next, we determine the probability $H_{\mathrm{OP}, \alpha, k^{\prime}}$ of no collision with user $k^{\prime}$, in region $\mathbf{S}_{\alpha}$. There are two different cases: user $k^{\prime}$ feeds back one level above $\alpha$ (i.e., one slot), or two levels, both above $\alpha$ (i.e., two slots). If user $k^{\prime}$ feeds back one level, the collision probability is $P_{\mathrm{c} 1}=\frac{1}{S_{\alpha}}$. If he feeds back two levels, then he picks two different slots among $S_{\alpha}$, resulting into a probability of collision of $P_{\mathrm{c} 2}=\frac{2}{S_{\alpha}}$ for $S_{\alpha} \neq 1$ (if $S_{\alpha}=$ 1 , only one level is reported and $P_{\mathrm{c} 2}=\frac{1}{S_{\alpha}}$ ). The probability of no collision with user $k^{\prime}$ averaged over $\bar{r}$ (equivalent for all users), can be written

$$
\begin{aligned}
H_{\mathrm{OP}, \alpha}= & H_{\mathrm{OP}, \alpha, k^{\prime}} \\
= & \sum_{j=1}^{M}\left(1-P_{\mathrm{c} 1} \Phi_{\mathrm{OP}, \alpha}\left(s=1 \mid \bar{r}_{j}\right)\right. \\
& \left.-P_{\mathrm{c} 2} \Phi_{\mathrm{OP}, \alpha}\left(s=2 \mid \bar{r}_{j}\right)\right) P\left(\bar{r}_{j}\right),
\end{aligned}
$$

where $\Phi_{\mathrm{OP}, \alpha}\left(s=1 \mid \bar{r}_{j}\right)$ and $\Phi_{\mathrm{OP}, \alpha}\left(s=2 \mid \bar{r}_{j}\right)$ are the conditional probabilities to feed back using one and two slots, respectively. First, we define $E_{\alpha, 1 s}\left(r_{a}, \bar{r}_{j}\right)$ the probability that $r_{a}$ is the best among all $N$ subchannels with $\bar{r}_{j}$. As subchannels are i.i.d. given $\bar{\gamma}$, the probability that rate $r_{a}$ is best can be written as the product over $N$ of the probability that each subchannel has $r_{i} \leq r_{a}$, given $\bar{\gamma}$. Then, integrating over the SNR region that gives $\bar{r}_{j}$, we get

$$
E_{\alpha, 1 s}\left(r_{a}, \bar{r}_{j}\right)=\int_{\frac{R}{\sigma_{j+1}^{1 / 3}}}^{\frac{R}{\sigma_{j}^{1 / 3}}}\left[\sum_{i=1}^{a} P\left(r_{i} \mid x_{k}\right)\right]^{N} p\left(x_{k}\right) d x_{k} .
$$

Summing up over the rates $r_{a} \geq \alpha \bar{r}_{j}$, and dividing by $P\left(\bar{r}_{j}\right)$, we get the conditional probability,

$$
\Phi_{\mathrm{OP}, \alpha}\left(s=1 \mid \bar{r}_{j}\right)=\frac{\sum_{a: r_{a} \geq \alpha \bar{r}_{j}} E_{\alpha, 1 s}\left(r_{a}, \bar{r}_{j}\right)}{P\left(\bar{r}_{j}\right)} .
$$

Similarly for $\Phi_{\mathrm{OP}, \alpha}\left(s=2 \mid \bar{r}_{j}\right)$, we have

$$
\begin{aligned}
\Phi_{\mathrm{OP}, \alpha}\left(s=2 \mid \bar{r}_{j}\right) & =\frac{\sum_{b: r_{b} \geq \alpha \bar{r}_{j}} \sum_{a: r_{a}>r_{b}} E_{\alpha, 2 s}\left(r_{a}, r_{b}, \bar{r}_{j}\right)}{P\left(\bar{r}_{j}\right)} \\
E_{\alpha, 2 s}\left(r_{a}, r_{b}, \bar{r}_{j}\right) & =\int_{\frac{R}{\sigma_{j+1}^{1 / 3}}}^{\frac{R}{\sigma_{j}^{1 / 3}}}\left(P\left(r_{a} \mid x_{k}\right)+\sum_{i=1}^{b} P\left(r_{i} \mid x_{k}\right)\right)^{N} \\
& \times p\left(x_{k}\right) d x_{k} .
\end{aligned}
$$

The derivations of $F_{\mathrm{OP}, \alpha, 1 \mathrm{~b}}, F_{\mathrm{OP}, \alpha, 2 \mathrm{~b}}, \Phi_{\mathrm{OP}, \alpha}\left(s=1 \mid \bar{r}_{j}\right)$ and $\Phi_{\mathrm{OP}, \alpha}\left(s=2 \mid \bar{r}_{j}\right)$ may seem rather tedious as they require counting out each case, due to the discrete nature of the variables. However, as the number of possible levels are limited, there are actually only a few cases. Moreover, we can determine beforehand the probability of occurrence of each discrete instantaneous rate/average rate pair $\left(r_{i}, \bar{r}_{j}\right)$. Table II shows the ratio value of each $\left(r_{i}, \bar{r}_{j}\right)$, whose probability 


$$
\begin{array}{r}
P\left(r_{i}, \bar{r}_{j}\right)=\int_{\bar{\gamma}=\sigma_{j}}^{\sigma_{j+1}} \int_{\gamma=\sigma_{i}}^{\sigma_{i+1}} \frac{1}{\bar{\gamma}} e^{-\frac{\gamma}{\bar{\gamma}}} d \gamma p(\bar{\gamma}) d \bar{\gamma}=\int_{R^{3} / \sigma_{j+1}}^{R^{3} / \sigma_{j}}\left(e^{-\frac{\sigma_{i} y}{R^{3}}}-e^{-\frac{\sigma_{i+1} y}{R^{3}}}\right) \frac{2}{3 R^{2}} y^{-1 / 3} d y, \\
P\left(r_{i}, \bar{r}_{j}\right)=\frac{2}{3} \Gamma\left(\frac{2}{3}\right) \sigma_{i}^{-\frac{2}{3}}\left[\gamma_{\mathrm{inc}}\left(\frac{\sigma_{i}}{\sigma_{j}}, \frac{2}{3}\right)-\gamma_{\mathrm{inc}}\left(\frac{\sigma_{i}}{\sigma_{j+1}}, \frac{2}{3}\right)\right]-\frac{2}{3} \Gamma\left(\frac{2}{3}\right) \sigma_{i+1}^{-\frac{2}{3}}\left[\gamma_{\mathrm{inc}}\left(\frac{\sigma_{i+1}}{\sigma_{j}}, \frac{2}{3}\right)-\gamma_{\mathrm{inc}}\left(\frac{\sigma_{i+1}}{\sigma_{j+1}}, \frac{2}{3}\right)\right] .
\end{array}
$$

is given by (23), with the change of variable $y=R^{3} / \bar{\gamma}$. Using similar derivations as previously, we get (24). If the probability of occurrence of a pair $P\left(r_{i}, \bar{r}_{j}\right)$ is zero or near zero, it is needless to count it as an event. Here we find that $P(4,1), P(5,1), P(5,2), P(5,3)$ and $P(1,5)$ are zero or near zero (in the order of $10^{-4}$ ). Thus, if $\alpha=2$, we only need to consider four cases for $F_{\mathrm{OP}, \alpha, 1 \mathrm{~b}}, \Phi_{\mathrm{OP}, \alpha}\left(s=1 \mid \bar{r}_{j}\right)$ and two cases for $F_{\mathrm{OP}, \alpha, 2 \mathrm{~b}}, \Phi_{\mathrm{OP}, \alpha}\left(s=2 \mid \bar{r}_{j}\right)$, and calculations are similar and systematic.

Finally, (17) becomes

$$
P_{\mathrm{OP}, \alpha, \mathrm{SR}}\left(\rho_{q}, r_{m}\right)=F_{\mathrm{OP}, \alpha}\left(\rho_{q}, r_{m}\right)\left(H_{\mathrm{OP}, \alpha}\right)^{K-1} .
$$

The joint probability that a user with ratio $\rho_{q}$ and rate $r_{m}$ is scheduled on subchannel $n, P_{\mathrm{OP}, \alpha}\left(\rho_{q}, r_{m}\right)$ is calculated by using the same approximation as for (13), where a user is allocated only if there is at least one user among $K$ who successfully fed back $\left(\rho_{q}, r_{m}\right)$ and, there is no other user who successfully fed back any other ratio strictly larger than $\rho_{q}$, or the same ratio with a rate larger than $r_{m}$, expressed as (25) on the next page, where $\sum_{q^{\prime}=q+1}^{Q} \sum_{i=1}^{M} P_{\mathrm{OP}, \alpha, \mathrm{SR}}\left(\rho_{q^{\prime}}, r_{i}\right)$ is the probability to feed back any ratio strictly larger than $\rho_{q}$ and $\sum_{i=m}^{M} P_{\mathrm{OP}, \alpha, \mathrm{SR}}\left(\rho_{q}, r_{i}\right)$, the probability to feed back the same ratio with a rate larger or equal to $r_{m}$. Let $P_{\mathrm{OP}, \alpha}\left(r_{m}\right)$ be the probability that a user with instantaneous rate $r_{m}$ is scheduled on subchannel $n$. Since the scheduled user can have any possible ratio larger than $\alpha$, but one occurrence of ratio/rate pair $\left(\rho_{q}, r_{m}\right)$ at a time, we have $P_{\mathrm{OP}, \alpha}\left(r_{m}\right)=$ $\sum_{q: \rho_{q} \geq \alpha} P_{\mathrm{OP}, \alpha}\left(\rho_{q}, r_{m}\right), \forall m \in\{1, \ldots, M\}$. Similar calculations for region $\mathbf{S}_{\beta}$ give the probability to schedule a user with rate $r_{m}, P_{\mathrm{OP}, \beta}\left(r_{m}\right), m=1, \ldots, M$, for each $\beta$. Finally, the throughput is approximated by

$$
\begin{aligned}
\tau_{\mathrm{OP}}\left(\alpha, \beta, S_{\alpha}\right) & =\sum_{m=1}^{M} r_{m} P_{\mathrm{OP}, \alpha}\left(r_{m}\right)+\sum_{m=1}^{M} r_{m} P_{\mathrm{OP}, \beta}\left(r_{m}\right) \\
& +r_{\text {rnd }} P_{\mathrm{OP}, \text { out }}
\end{aligned}
$$

for a fixed number of users $K$. This expression enables to determine the optimal parameter values $\alpha, \beta, S_{\alpha}$ that maximize the throughput (see Section VI-B).

\section{B. Analysis for Full CSI RA Feedback}

In this case, as any user feeds back with probability 1 , for $\bar{r}_{j}=\frac{\rho_{q}}{r_{m}}$, we have $P_{\mathrm{FP}, \mathrm{SR}}\left(\rho_{q}, r_{m}\right)=P\left(r_{m}, \bar{r}_{j}\right) \times$ $\left(1-\frac{1}{S}\right)^{K-1}$. The joint probability that a user with ratio $\rho_{q}$ and rate $r_{m}$ is scheduled on subchannel $n, P_{\mathrm{FP}}\left(\rho_{q}, r_{m}\right)$, can be determined similarly as in (25), so that, denoting $P_{\mathrm{FP}}\left(r_{m}\right)=$ $\sum_{q=1}^{Q} P_{\mathrm{FP}}\left(\rho_{q}, r_{m}\right)$, the approximated throughput becomes

$$
\tau_{\mathrm{FP}}=\sum_{m=1}^{M} r_{m} P_{\mathrm{FP}}\left(r_{m}\right)+r_{\mathrm{rnd}}\left(1-\sum_{m=1}^{M} P_{\mathrm{FP}}\left(r_{m}\right)\right) .
$$

\section{Analysis for Threshold CSI RA Feedback}

In this scheme, users having subchannels whose ratios are greater than or equal to a threshold $\delta$ make a feedback by selecting one slot randomly. For $\rho_{q} \geq \delta$ and $\bar{r}_{j}=\frac{\rho_{q}}{r_{m}}$, the probability of successful feedback for a user is $P_{\mathrm{TP}, \delta, \mathrm{SR}}\left(\rho_{q}, r_{m}\right)=$ $P\left(r_{m}, \bar{r}_{j}\right) \times\left(H_{\mathrm{TP}, \delta}\right)^{K-1}, H_{\mathrm{TP}, \delta}$ denoting the probability of no collision with a competitor. As a competitor feeds back if at least one of his subchannels has a ratio larger than $\delta$, we have $H_{\mathrm{TP}, \delta}=\sum_{i=1}^{M}\left(1-\frac{\Phi_{\mathrm{TP}, \delta}\left(s=1 \mid \bar{r}_{i}\right)}{S}\right) P\left(\bar{r}_{i}\right)$, where the conditional probability of having a feedback given the average rate $\Phi_{\mathrm{TP}, \delta}\left(s=1 \mid \bar{r}_{i}\right)$ is equal to one minus the probability that all subchannels are strictly below $\delta, P_{\forall n}\left(\rho<\delta \mid \bar{r}_{i}\right)$. For a certain $\bar{r}_{i}$, denoting $l^{*}$ the largest index such that $r_{l}<\delta \bar{r}_{i}$, this can be determined by

$$
\begin{aligned}
P_{\forall n}\left(\rho<\delta \mid \bar{r}_{i}\right) & =\int_{\sigma_{i+1}}^{\sigma_{i}}\left(\int_{1}^{\sigma_{l^{*}+1}} \frac{1}{\bar{\gamma}} e^{-\frac{\gamma}{\bar{\gamma}}} d \gamma\right)^{N} \\
& \times \frac{1}{P\left(\bar{r}_{i}\right)} p(\bar{\gamma}) d \bar{\gamma}
\end{aligned}
$$

which can be solved with similar derivations as in Section V-A. Denoting $P_{\mathrm{TP}, \delta}\left(\rho_{q}, r_{m}\right)$ the joint probability that a user with ratio $\rho_{q}$ and rate $r_{m}$ is scheduled on subchannel $n$, determined similarly as in (25), we obtain the approximated throughput as a function of the threshold $\delta$,

$$
\tau_{\mathrm{TP}}(\delta)=\sum_{m=1}^{M} r_{m} P_{\mathrm{TP}, \delta}\left(r_{m}\right)+r_{\mathrm{rnd}}\left(1-\sum_{m=1}^{M} P_{\mathrm{TP}, \delta}\left(r_{m}\right)\right),
$$

where $P_{\mathrm{TP}, \delta}\left(r_{m}\right)=\sum_{q: \rho_{q} \geq \delta} P_{\mathrm{TP}, \delta}\left(\rho_{q}, r_{m}\right)$, and the optimal threshold can be easily computed as

$$
\delta_{\text {opt }}=\arg \max _{\delta \in \mathcal{Q}} \tau_{\mathrm{TP}}(\delta) .
$$

\section{NuMERICAL RESUlTS}

Simulations are performed for $N=8$ subchannels with the channel models in Section IV and cell radius $R=1000 \mathrm{~m}$. As $N$ grows, the gap between overheads $B_{\mathrm{TRA}}$ and $B_{\mathrm{OMax}}$ increases (for $N=8, B_{\mathrm{TRA}} / B_{\mathrm{OMax}} \simeq 1.2$ but for $N=$ $32, B_{\mathrm{TRA}} / B_{\mathrm{OMax}} \simeq 1.6$ ), so the performance gain of the proposed scheme will increase. There are $S=20$ feedback 


$$
\begin{aligned}
& P_{\mathrm{OP}, \alpha}\left(\rho_{q}, r_{m}\right)=\left(1-\sum_{q^{\prime}=q+1}^{Q} \sum_{i=1}^{M} P_{\mathrm{OP}, \alpha, \mathrm{SR}}\left(\rho_{q^{\prime}}, r_{i}\right)-\sum_{i=m}^{M} P_{\mathrm{OP}, \alpha, \mathrm{SR}}\left(\rho_{q}, r_{i}\right)+P_{\mathrm{OP}, \alpha, \mathrm{SR}}\left(\rho_{q}, r_{m}\right)\right)^{K} \\
& -\left(1-\sum_{q^{\prime}=q+1}^{Q} \sum_{i=1}^{M} P_{\mathrm{OP}, \alpha, \mathrm{SR}}\left(\rho_{q^{\prime}}, r_{i}\right)-\sum_{i=m}^{M} P_{\mathrm{OP}, \alpha, \mathrm{SR}}\left(\rho_{q}, r_{i}\right)\right)^{K}
\end{aligned}
$$

slots, $L=2, b_{\mathrm{ID}}=10$ bits and $b_{\mathrm{CRC}}=8$ bits. In the simulations, we assume a 5-tap Rayleigh fading model for generating channel frequency responses with correlated gains ${ }^{8}$.

\section{A. OMax Algorithm}

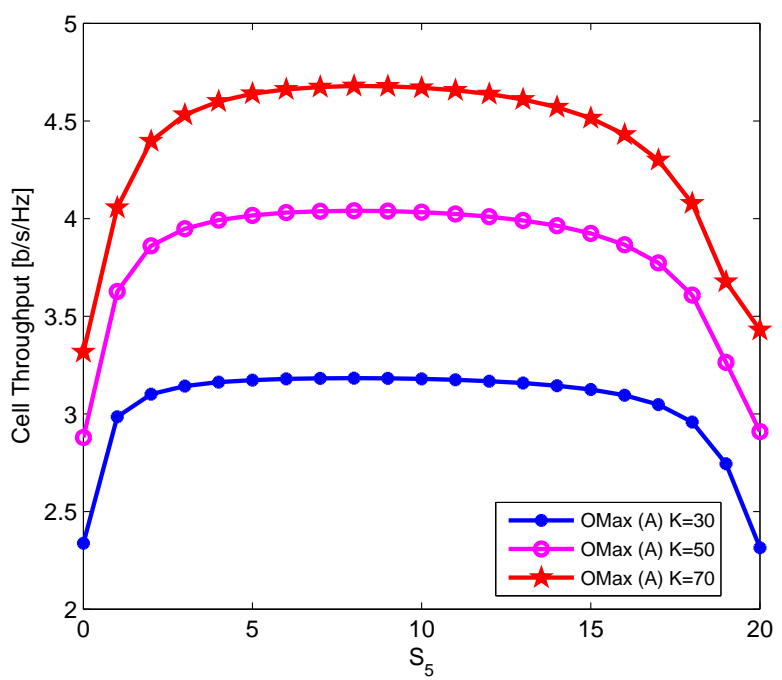

Fig. 2. Max CSI algorithm with orthogonal RA slots (OMax), analysis

Fig. 2 shows the analytical throughput for the OMax scheme, for all possible numbers of slots $S_{5}$ and different number of users. We observe that there is not a distinct optimal point, even though the maximum occurs at $S_{5}=9$ in all cases. That is, by an equal partitioning of slots among the two layers, a near optimal throughput is achieved. As the number of users grows, the flatness of the curve diminishes slowly. Note that equal slot distribution results into unequal collision probabilities of the two layers as the probability of occurrence of $r_{5}$ is smaller than that of $r_{4}$, as $\gamma$ follows an exponential distribution. A lower collision probability is achieved for the feedback of the highest quality CSI, hence providing a higher protection.

Fig. 3 compares the cell throughput obtained by analysis (A) and simulations (S). Fig. 4 shows the net cell throughput $\tilde{\tau}$, i.e., the cell throughput given the overhead used for UL CSI defined as $\tilde{\tau}=\tau \times \frac{b_{\text {data }}}{b_{\text {data }}+b_{\mathrm{OH}}}$, measuring the throughputoverhead trade-off of the different schemes, with $\tau$ the cell throughput, $b_{\text {data }}$ the number of bits carrying data assuming

\footnotetext{
${ }^{8}$ Unlike in the analysis, users are prohibited within a circle of radius $1.5 \mathrm{~m}$ around the center to avoid extreme values of SNR.
}

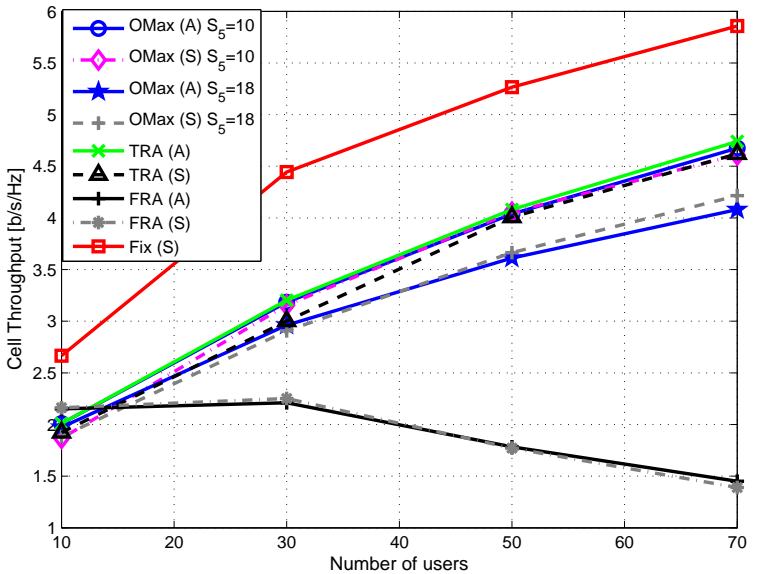

Fig. 3. Cell throughput for Max CSI algorithm, analysis (A) and simulation (S)

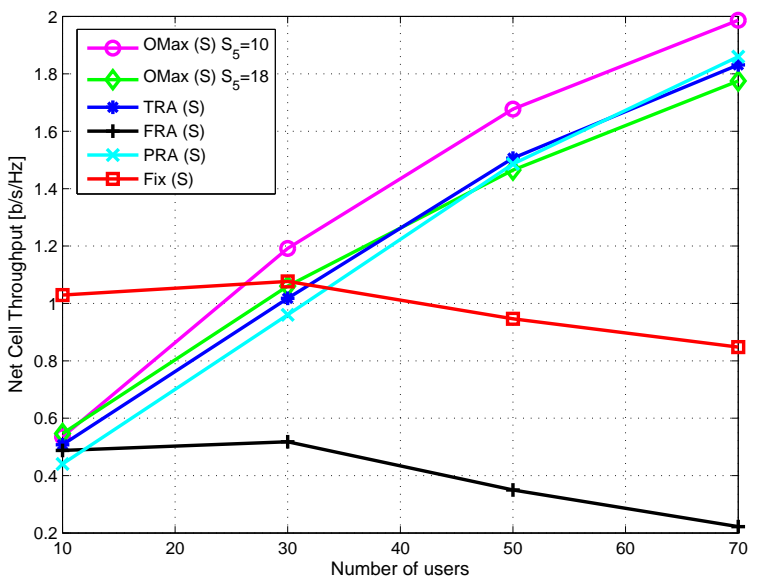

Fig. 4. Net Cell throughput for Max CSI algorithm

$10 \mathrm{MC}$ symbols per frame and $b_{\mathrm{OH}}$, the number of overhead bits for CSI given the feedback scheme. In addition to the reference schemes in Section III, we define the throughput optimal Full CSI-Fixed (Fix) scheme where all users feed back their full CSI by sequential feedback, and the Per-Subchannel $R A$ (PRA) scheme based on [16], where $S_{\text {Sub }}$ RA slots are allocated per subchannel. If a user has a CSI higher than the threshold, he feeds it back by randomly selecting one slot among $S_{\text {Sub }}$. We set $S_{\text {Sub }}=5$ as in [16], providing a good throughput-overhead trade-off. This process is repeated for each subchannel. For fair comparison, we set to 1 the access 
probability for all users with CSI to report ${ }^{9}$. Fix and $P R A$ schemes use $B_{\mathrm{Fix}}=K\left(b_{\mathrm{ID}}+\left\lceil\log _{2}(M)\right\rceil \times N+b_{\mathrm{CRC}}\right)$ and $B_{\mathrm{PRA}}=S_{\text {Sub }} N\left(b_{\mathrm{ID}}+\left\lceil\log _{2}(L)\right\rceil+b_{\mathrm{CRC}}\right)$ bits.

Fig. 3 shows that in all cases, simulations validate the analysis well. For a low number of users, the FRA scheme achieves a higher throughput than OMax, but is outperformed as the number of users increases, due to more collisions. In terms of throughput, OMax with a near-optimal slot distribution $\left(S_{5}=S_{4}=10\right)$ achieves a similar performance as the $T R A$, even with the increased number of collisions due to layering, as explained in Section III. That is, the drawback due to the higher number of collisions is canceled out by the variable collision protection effect, i.e., the feedback of a whole information using one slot is in this case similar to the feedback of parts of that information using multiple slots but with a higher protection of the important parts. Then, as shown in Fig. 4, the difference between both schemes comes from the overhead. The proposed scheme outperforms all reference schemes, for $S_{5}=S_{4}=10$. With a different slot distribution $S_{5}=18$, performance degrades notably for $K=70$. The net throughput for Fix scheme is largely decreased due to overhead, and number of collisions for FRA. Finally, we observe that both reference schemes $P R A$ with persubchannel feedback and TRA with grouped feedback have a similar net throughput and are outperformed by OMax with $S_{5}=S_{4}=10$. But the performance of $P R A$ may degrade with more subchannels, due to the linear increase of $B_{\mathrm{PRA}}$ with $N$

\section{B. OPFS Algorithm}

To maximize the throughput (26), we need to find the optimal values of three variables,

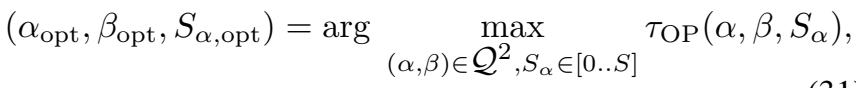

which may vary with $K$. For this, the discrete nature of the problem requires the calculation of all the possible combinations of $\alpha, \beta, S_{\alpha}$, and determining the one giving the highest throughput. It may seem to be a rather complex combinatorial problem, but the search space can be drastically reduced with the following observations. As we assume that the BS has knowledge of the average rates of each user, the search for the optimal thresholds $\alpha, \beta$ can be restricted to values strictly larger than one, since the scheduler is only interested in ratios $\rho$ that are strictly larger than one. Here, this corresponds to setting the minimal value of $\beta$ to $4 / 3$. Furthermore, the threshold values where probabilities of having $(\rho, r)$ are zero may not be considered, since it wouldn't make sense to prepare some feedback slots for CSI values that never or almost never occur. Thus, the maximal value of $\alpha$ is set to 2 . This results into only 3 possible combinations: $(\alpha, \beta)=(2,4 / 3),(2,3 / 2)$ and $(3 / 2,4 / 3)$. Then, for each pair, the optimal value of the number of slots $S_{\alpha, \text { opt }}$ can be easily obtained, for each $K$.

\footnotetext{
${ }^{9}$ Including access probabilities in our scheme may lead to even larger improvement, by setting different access probabilities to the priority levels. While this issue is out of the scope of the paper, it is an interesting direction for future work.
}

Finally, the comparison of the throughput values at $S_{\alpha, \text { opt }}$ for each of the three pairs gives the optimal throughput, for each $K$. Actually, we found that $S_{\alpha, \text { opt }}$ was mostly constant over $K$ (for $(\alpha, \beta)=(2,4 / 3), S_{\alpha, \text { opt }}=16$ for $K=10,30$ and $S_{\alpha, \text { opt }}=15$ for $K=50,70$; for $(\alpha, \beta)=(2,3 / 2)$, $S_{\alpha, \mathrm{opt}}=19$ except for $K=70$ where $S_{\alpha, \mathrm{opt}}=18$ and for $(\alpha, \beta)=(3 / 2,4 / 3), S_{\alpha, \text { opt }}=17$ for all $\left.K\right)$. This is because the proportion of users in $\mathbf{S}_{\alpha}$ and $\mathbf{S}_{\beta}$ remains constant with $K$, and so does the slots distribution. Thus, we can assume that $S_{\alpha, \text { opt }}$ is constant over $K$. The analytical throughput for the three combinations above is shown in Fig. 5. We can conclude that the optimal set of values are $\left(\alpha_{\text {opt }}, \beta_{\text {opt }}, S_{\alpha, \text { opt }}\right)=(2,4 / 3,16)$. Likewise, the optimization of the throughput of TRA scheme in (30) gives $\delta_{\mathrm{opt}}=4 / 3$.

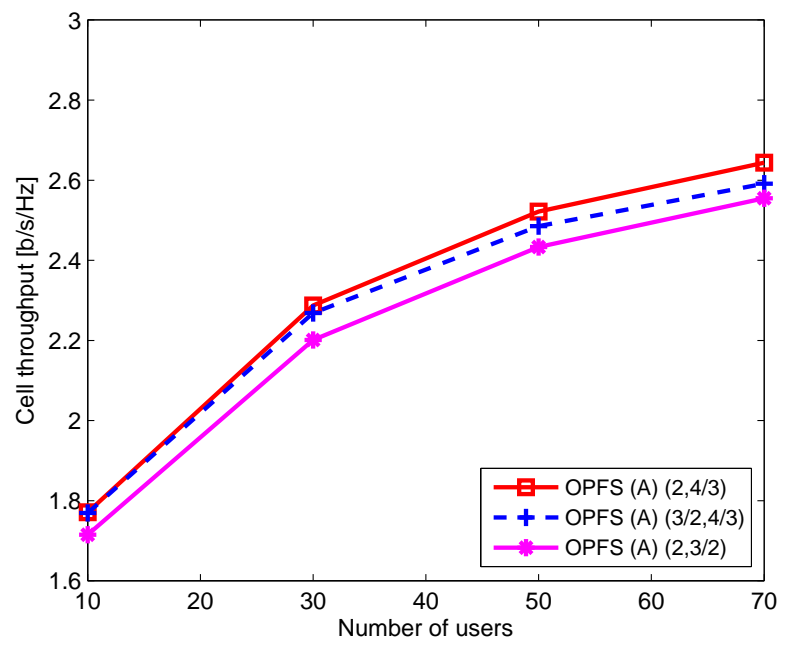

Fig. 5. Cell throughput for PFS algorithm for different $(\alpha, \beta)$ with the optimal $S \alpha$ in each case

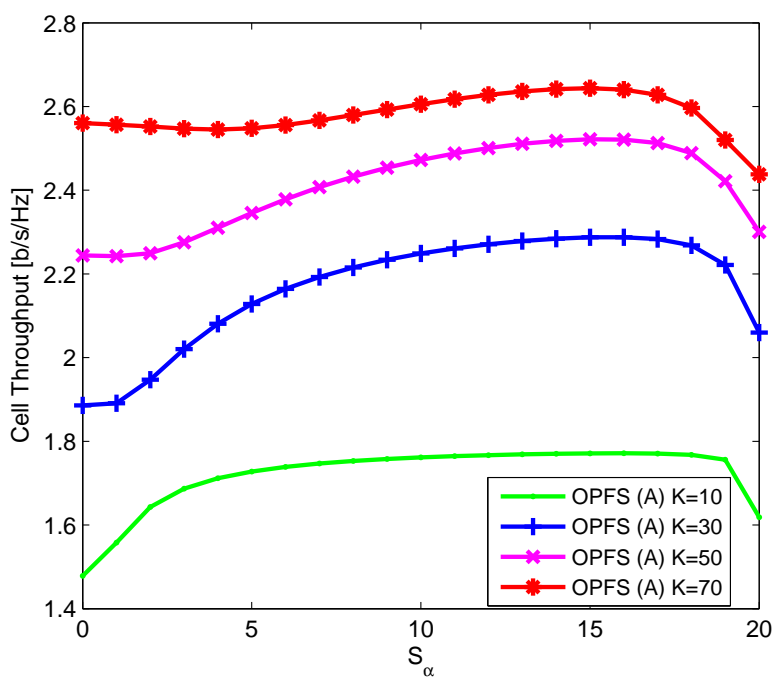

Fig. 6. Cell throughput for PFS algorithm with orthogonal RA slots (OPFS), analysis

Next, Fig. 6 shows how the OPFS analytical throughput scales with the numbers of slots $S_{\alpha}$ and number of users $K$. 


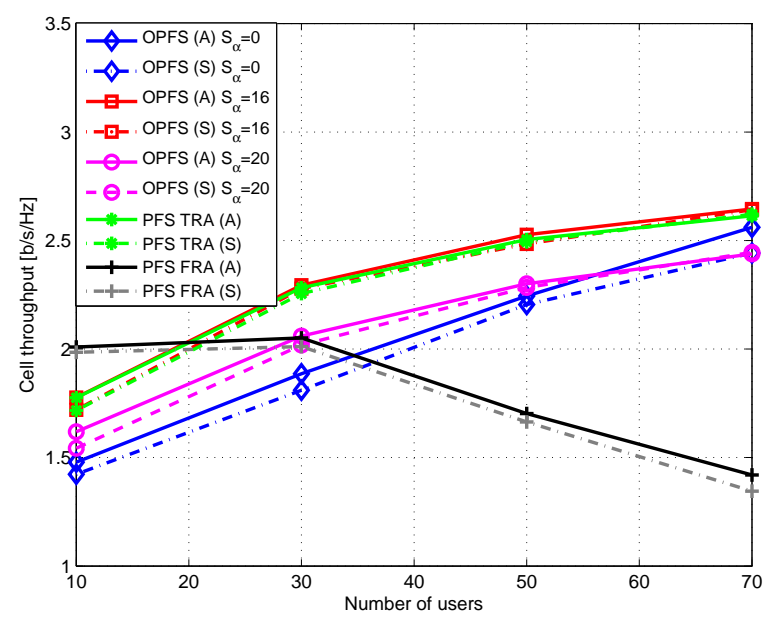

Fig. 7. Cell throughput for PFS algorithm, analysis (A) and simulation (S)

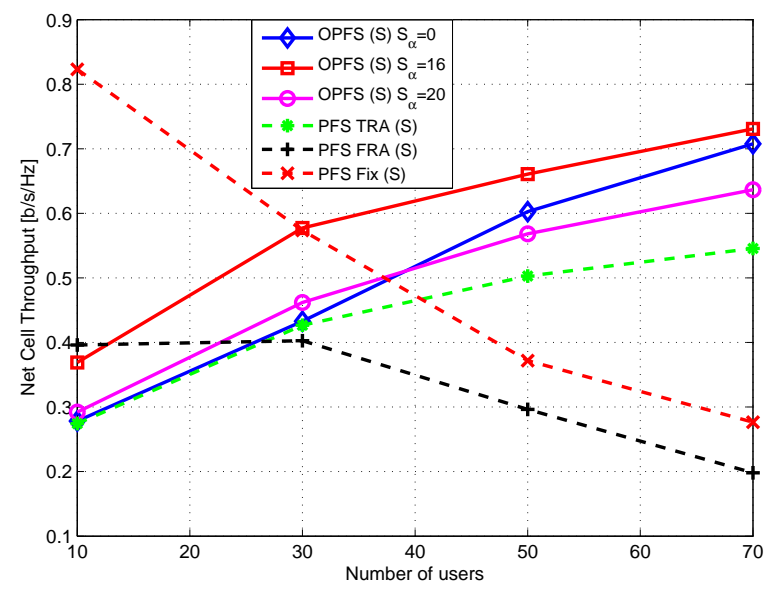

Fig. 8. Net cell throughput for PFS algorithm

This time, we observe a larger variation of the throughput against the number of slots. In Fig. 7, the throughput of OPFS for $S_{\alpha}=0,16,20$ and the 3 reference schemes are shown. In all cases, simulation and analysis match closely. The simulated throughput is slightly lower than the analytical one, as in the simulations we consider decoding errors at the MS receiver, unlike in the analysis. Again, the throughput of FRA is degraded as the number of users grows, due to the high number of collisions. The performance achieved by the proposed method varies notably for different $S_{\alpha}$. We can see also that $O P F S$ with $S_{\alpha, \text { opt }}$ globally achieves a similar performance as TRA, even with the increased number of collisions due to layering. That is, a higher collision protection is achieved for the feedback in the $\mathbf{S}_{\alpha}$ region, at the expense of lower protection in $\mathbf{S}_{\beta}$ region, while ensuring the same overall performance as TRA.

Next, the net throughput and fairness performance simulations are given by Figs. 8 and 9, respectively. As the fairness metric, Jain's index $J$ is introduced, defined as [24] $J=\frac{\left(\sum_{k=1}^{K} R_{k}\right)^{2}}{K \times \sum_{k=1}^{K} R_{k}^{2}}$, where $J=1$ for perfect fairness. Again the

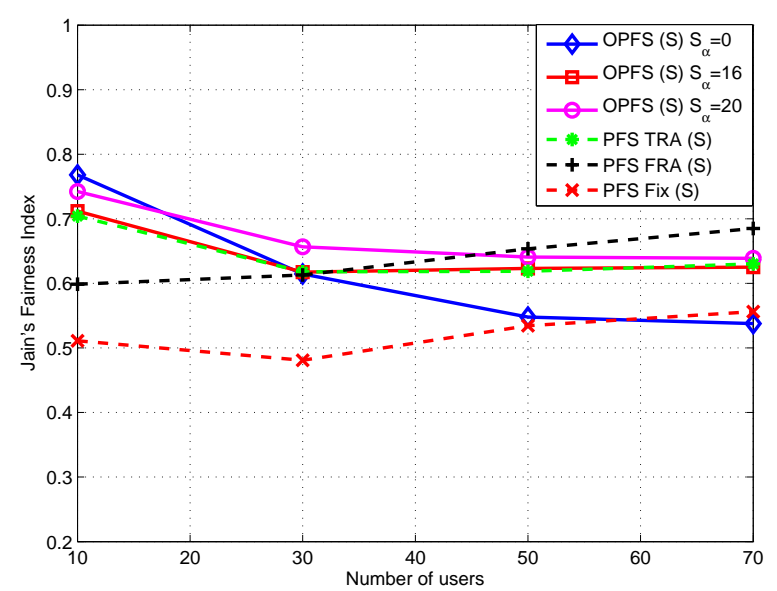

Fig. 9. Jain's fairness index for PFS algorithm

reference Fix and FRA offer extremely poor net throughput due to overhead and collisions, respectively. At the same time, it may seem surprising that Fix has a relatively low fairness, but recall that this scheme is optimal in terms of proportional fairness, which does not necessarily mean equal rate allocation among users. Thus, FRA achieves a high Jain's metric performance as the high number of collisions implies that users are allocated randomly. Compared to TRA, our proposed scheme achieves a higher net throughput for all values of $S_{\alpha}$, while the largest gap is observed for $S_{\alpha}=16$. Good fairness performance is achieved by choosing $S_{\alpha}=20$ and $S_{\alpha}=16$, the latter ensuring the same level as TRA. Overall, OPFS with $S_{\alpha}=16$ provides the best performance, when net throughput and fairness are jointly considered.

Finally, we evaluate our proposed OPFS scheme for the case with heterogeneous users. We consider that half of the users, referred as Best Effort (BE) are not delay sensitive, while the other half, referred as Real Time (RT) are delay sensitive. We assume for OPFS that the RT users are allowed to feed back both their best and second best levels (regions $\mathbf{S}_{\alpha}$ and $\mathbf{S}_{\beta}$ ), while the BE users are only allowed to feed back their best levels $\left(\mathbf{S}_{\alpha}\right)$. By contrast, TRA and FRA schemes do not benefit from such service differentiation, as, e.g. in $T R A$, one slot is used whether the user feeds back both levels or only the best. Therefore, TRA and FRA work similarly as before, for both types of users. Table III shows the outage probability of RT users for $K=30$, where delay and user rate are both taken into account by defining a user to be in outage if his short-term rate averaged every 10 frames is below a reference rate $R_{\text {Ref }}=0.2 \mathrm{~b} / \mathrm{s} / \mathrm{Hz}$. We observe that $O P F S$ with $S_{\alpha}=6$ and 2 both largely outperform $T R A$ and $F R A$, thanks to the reduced collisions as BE users are only allowed to feed back in $\mathbf{S}_{\alpha}$ while $\mathbf{S}_{\beta}$ is reserved for RT users with second best levels, whereas all users with best and/or second best levels feedback in TRA (and all users in FRA). Table III also compares the effect of such differentiation on the total net throughput, which, as expected, decreases for $O P F S$ as a new QoS constraint is introduced. However, we observe that $O P F S$ with $S_{\alpha}=6$ outperforms TRA both in 
net throughput and outage, while $O P F S$ with $S_{\alpha}=2$ further decreases outage but at the expense of throughput. Thus, OPFS enables to decrease outage for RT users, even though service differentiation only occurs during CSI feedback. We can think that the proposed feedback scheme may bring further benefits when combined with some more sophisticated schedulers with QoS differentiation.

TABLE III

Outage Probability AND Net Throughput PERFormance With HETEROGENEOUS USERS FOR $K=30$.

\begin{tabular}{|l|c|c|c|c|}
\hline Scheme & PFS TRA & OPFS $S_{\alpha}=6$ & OPFS $S_{\alpha}=2$ & PFS FRA \\
\hline $\begin{array}{l}\text { RT users' } \\
\text { outage } \\
\text { probability }\end{array}$ & 0.27 & 0.17 & 0.09 & 0.6 \\
\hline $\begin{array}{l}\text { Net } \\
\text { throughput } \\
\text { with Serv. }\end{array}$ & 0.42 & 0.44 & 0.34 & 0.4 \\
$\begin{array}{l}\text { Diff. } \\
\text { [b/s/Hz] }\end{array}$ & 0.42 & 0.5 & 0.45 & 0.4 \\
\hline $\begin{array}{l}\text { Net } \\
\text { throughput } \\
\text { without }\end{array}$ & & & \\
$\begin{array}{l}\text { Serv. Diff. } \\
\text { [b/s/Hz] }\end{array}$ & & & \\
\hline
\end{tabular}

\section{CONCLUSION}

We have proposed a method for contention-based CSI feedback in an UL control channel for DL scheduling of a MC system, with orthogonal partition among the CSIs of different quality levels. The scheme provides a variable collision protection depending on the importance of the CSI to feed back, while reducing the feedback overhead. The analysis, which assumed discrete random variables due to the discrete AM model, provided a novel method for assessing scheduling performance, which can be used generally for various problems. The analytical and simulation results had an excellent match, and showed that, with adequate slot distributions, the proposed scheme achieved the best net throughput/fairness performance for Max CSI and PFS compared to conventional reporting schemes, thanks to its ability to prioritize the best quality CSI and reducing collisions, thus maximizing the multi-user diversity gain at the scheduler.

Different extensions may be considered, such as analysis with more elaborate channel models, or multiple user priority classes from higher level requirements, and multi-antenna systems.

\section{ACKNOWLEDGMENT}

This work was supported by the Grants-in-Aid for JSPS Fellow no. 204205, for Scientific Research no. 21760289 and no. 10595739 from the Ministry of Education, Science, Sports, and Culture of Japan.

\section{REFERENCES}

[1] R. Knopp and P. Humblet, "Information capacity and power control in single cell multiuser communications," in Proc. IEEE ICC, vol. 1, Seattle, WA, June 1995, pp. 331-335.
[2] W. Wang, T. Ottosson, M. Sternad, A. Ahlen and A. Svensson, "Impact of multiuser diversity and channel variability on adaptive OFDM," in Proc. IEEE VTC-Fall, vol. 1, Orlando, FL, October 2003, pp. 547-551.

[3] J. Gross, H.-F. Geerdes, H. Karl and A. Wolisz, "Performance Analysis of Dynamic OFDMA Systems with Inband Signaling," IEEE Journal on Selec. Areas in Comm., vol. 24, no. 3, pp. 427-436, March 2006.

[4] R. Agarwal, R. Vannithamby and J.M. Cioffi, "Optimal Allocation of Feedback Bits for Downlink OFDMA Systems," in Proc. IEEE ISIT, Toronto, Canada, July 2008, pp. 1686-1690.

[5] D. Gesbert and M.S. Alouini, "How Much Feedback is Multi-User Diversity Really Worth," in Proc. ICC, vol. 1, June 2004, pp. 234-238.

[6] Z.H. Han and Y.H. Lee, "Opportunistic scheduling with partial channel information in OFDMA/FDD systems," in Proc. IEEE VTC-Fall, vol. 1, September 2004, pp. 511-514.

[7] S. Sanayei and A. Nosratinia, "Opportunistic Downlink Transmission with Limited Feedback," IEEE Trans. Info. Theory, vol. 53, no. 11, pp. 4363-4372, November 2007.

[8] O. Somekh, O. Simeone, A.M. Haimovich and Y. Bar-Ness, "Sum-Rate Analysis of General OFDM Downlink Channels with 1-bit Feedback per Sub-Carrier," in Proc. IEEE CISS, Princeton, NJ, March 2006.

[9] M. Kaneko, P. Popovski and H. Yomo, "Adaptive Provision of CSI Feedback in OFDMA Systems," in Proc. IEEE PIMRC, Helsinki, Finland, September 2006, pp. 1-5.

[10] J. Leinonen, J. Hämäläinen and M. Juntti, "Performance Analysis of Downlink OFDMA Resource Allocation with Limited Feedback," IEEE Trans. Wireless Comm., vol. 8, no. 6, pp. 2927-2937, June 2009.

[11] T. Tang and R.W. Heath, "Opportunistic feedback for downlink multiuser diversity," IEEE Commun. Letters, vol. 9, no. 10, pp. 948-950, October 2005.

[12] S. Patil and G. de Veciana, "Reducing Feedback for Opportunistic Scheduling in Wireless Systems," IEEE Trans. Wireless Comm., vol. 6, no. 12, pp. 4227-4232, December 2007.

[13] Y.S. Al-Harthi, A.H. Tewfik and M.S. Alouini, "Multiuser Diversity with Quantized Feedback," IEEE Trans. Wireless Comm., vol. 6, no. 1, pp. 330-337, January 2007.

[14] V. Hassel, D. Gesbert, M.S. Alouini and G.E. Øien, "A Threshold-Based Channel State Feedback Algorithm for Modern Cellular Systems," IEEE Trans. Wireless Comm., vol. 6, no. 7, pp. 2422-2426, July 2007.

[15] J. So, "Opportunistic feedback with Multiple Classes in Wireless Systems," IEEE Commun. Letters, vol. 13, no. 6, pp. 384-386, June 2009.

[16] T. Tang, R.W. Heath Jr., S. Cho and S. Yun, "Opportunistic Feedback in Clustered OFDM Systems," in Proc. WPMC, San Diego, CA, September 2006.

[17] R. Agarwal, V. Majjigi, Z. Han, R. Vannithamby and J. Cioffi, "Low Complexity Resource Allocation with Opportunistic Feedback over Downlink OFDMA Networks," IEEE Journal on Selec. Areas in Comm., vol. 26, no. 8, pp. 1462-1472, October 2008.

[18] J. Chen, R.A. Berry and M.L. Honig, "Limited Feedback Schemes for Downlink OFDMA Based on Sub-Channel Groups," IEEE Journal on Selec. Areas in Comm., vol. 26, no. 8, pp. 1451-1461, October 2008.

[19] M. Kaneko, K. Hayashi, P. Popovski, H. Yomo and H. Sakai, "Uplink Random Access Scheme with Prioritized Orthogonal Layers for OFDMA CSI Feedback," in Proc. IEEE Globecom, Honolulu, Hawai, Nov-Dec 2009.

[20] — "Uplink Random Access Scheme with Variable Collision Protection for OFDMA CSI Feedback," in Proc. WPMC, Sendai, Japan, September 2009.

[21] A. Kühne and A. Klein, "Throughput Analysis of Multi-user OFDMASystems using Imperfect CQI Feedback and Diversity Techniques," IEEE Journal on Selec. Areas in Comm., vol. 26, no. 8, pp. 1440-1450, October 2008

[22] S. T. Chung and A. J. Goldsmith, "Degrees of Freedom in Adaptive Modulation: A Unified View," IEEE Trans. Comm., vol. 49, no. 9, pp. 1561-1571, September 2001.

[23] I. Gradshteyn and I. Ryzhik, Table of Integrals, Series and Products. San Diego, CA: Academic Press, 2000.

[24] R. Jain, The art of computer systems performance analysis. John Wiley and Sons, 1991. 


\begin{tabular}{|c|} 
\\
\\
PLACE \\
PHOTO \\
HERE \\
\end{tabular}

Megumi Kaneko received her B.S. and MSc. degrees in communication engineering in 2003 and 2004 from Institut National des Télécommunications (INT), France, jointly with a MSc. from Aalborg University, Denmark, where she received her Ph.D. degree in 2007. From January to July 2007, she was a visiting researcher in Kyoto University, Kyoto, Japan, and a JSPS post-doctoral fellow from April 2008 to August 2010. She is currently an Assistant Professor in the Department of Systems Science, Graduate School of Informatics, Kyoto University. Her research interests include wireless communication, protocol design and communication theory. She received the 2009 Ericsson Young Scientist Award, the IEEE Globecom'09 Best Paper Award in Wireless Communications Symposium and the 2011 Funai Young Researcher's Award.

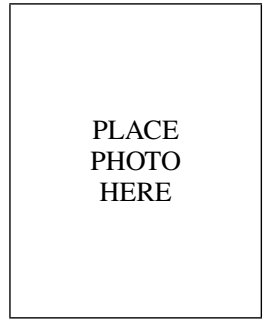

Hiroyuki Yomo received the B.S., M.S., and Ph.D. degrees in communication engineering from Osaka University, Japan, in 1997, 1999 and 2002, respectively. From April 2002 to March 2004, he was a Post-doctoral Fellow at Aalborg University, Denmark. From April 2004 to September 2004, he was at NEC Corporation, Japan. In October 2004, he joined Aalborg University, Denmark, as an Assistant Research Professor, and worked as an Associate Professor from February 2006 to March 2008. From April 2008 to March 2010, he was a senior researcher at ATR, Japan. Since April 2010, he has been an Associate Professor at Kansai University, Japan. He is also affiliated with Aalborg University as an Assigned Associate Professor and with ATR as a guest researcher. He received the 2010 Funai Academic Award from Funai Foundation for Information Technology. He has received several best paper awards, including the one at IEEE Globecom 2009. His main research interests are access technologies, radio resource management, and link-layer techniques in the area of ad hoc/vehicular network, wireless LAN, cellular system, cognitive radio, and wireless network coding. He is an editor of IEEE Communications Letters.

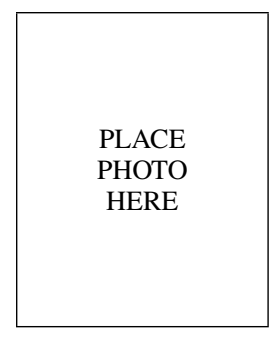

Kazunori Hayashi received the B.E., M.E. and $\mathrm{Ph} . \mathrm{D}$. degrees in communication engineering from Osaka University, Osaka, Japan, in 1997, 1999 and 2002 , respectively. Since 2002, he has been with the Department of Systems Science, Graduate School of Informatics, Kyoto University. He is currently an Associate Professor there. His research interests include digital signal processing for communication systems. He received the ICF Research Award from the KDDI Foundation in 2008, the IEEE Globecom 2009 Best Paper Award, and the IEICE Communications Society Excellent Paper Award in 2011.

\begin{tabular}{|c|}
\hline \\
\\
PLACE \\
PHOTO \\
HERE \\
\end{tabular}

Petar Popovski received the Dipl.-Ing. in electrical engineering and Mr.-Ing. in communication engineering from Sts. Cyril and Methodius University, Skopje, Macedonia, in 1997 and 2000, respectively and Ph. D. from Aalborg University, Denmark, in 2004. He was Assistant Professor at Aalborg University from 2004 to 2009 . From 2008 to 2009 he held part-time position as a wireless architect at Oticon A/S. Since 2009 he is an Associate Professor at Aalborg University. He has more than 100 publications in journals, conference proceedings and books and has more than 25 patents and patent applications. He has received the Young Elite Researcher award from the Danish Free Research Council. He has received several best paper awards, including the ones from IEEE Globecom and IEEE Communication Theory Workshop. Prof. Popovski serves on the editorial board of several journals, including IEEE Transactions on Wireless Communications and IEEE Communications Letters. His research interests are in the broad area of wireless communication and networking, information theory and protocol design.

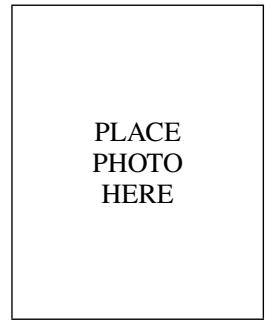

Hideaki Sakai received the B.E. and D.E. degrees in applied mathematics and physics from Kyoto University, Kyoto, Japan, in 1972 and 1981, respectively. From 1975 to 1978 , he was with Tokushima University. He is currently a Professor in the Department of Systems Science, Graduate School of Informatics, Kyoto University. He spent 6 months from 1987 to 1988 at Stanford University as a Visiting Scholar. His research interests are in the areas of adaptive and statistical signal processing. He served as an associate editor of IEEE Trans. Signal Processing from Jan. 1999 to Jan. 2001. He is a Fellow of IEEE. 